\title{
Long-term outcomes of incidental gallbladder carcinoma without additional resection: A single institution experiment
}

\author{
TOMOHIRO SUGIYAMA ${ }^{1}$, KENTA MAKINO ${ }^{1}$, YUKIKO FUKUI ${ }^{1}$, HIROMITSU KINOSHITA ${ }^{1}$, \\ AKIRA MIKI $^{1}$, SHIGEKI UCHIDA ${ }^{1}$, MICHIHIKO TSUBONO ${ }^{1}$ and YASUSHI ADACHI ${ }^{2}$ \\ Departments of ${ }^{1}$ Surgery and ${ }^{2}$ Clinical Pathology, Toyooka Hospital, Toyooka, Hyogo 668-0065, Japan
}

Received April 10, 2019; Accepted April 22, 2020

DOI: $10.3892 / \mathrm{mco} .2020 .2054$

\begin{abstract}
Incidental gallbladder carcinoma (IGC), defined as unexpected malignancy identified in the surgical gallbladder specimen of a cholecystectomy performed for a benign diagnosis, can be difficult to suspect preoperatively. Furthermore, there are valid clinical reasons to defer reoperation for additional resection, particularly in elderly patients. The present study aimed to determine the long-term outcomes and prognostic factors associated with recurrence in patients with IGC. The medical records of 678 patients who underwent cholecystectomy at Toyooka Hospital between September 2011 and November 2017 were reviewed. The cases identified to be IGC were retrospectively analyzed to determine patient and histopathological characteristics, surgical details, long-term outcomes and factors associated with cancer recurrence. A total of 22 patients were diagnosed with gallbladder carcinoma following cholecystectomy by histopathological examination, and 12 of these were identified to be IGC. The median age was 80 years (range 70-89 years). Although 6 of the 12 patients with IGC had stage pT2 or pT3 tumors, only 1 patient underwent additional resection. Recurrence occurred in 3 of the 8 patients who did not undergo additional resection and were available for long-term follow-up. Recurrence was not associated with the extent of tumor invasion but may be associated with other histopathological findings, preoperative
\end{abstract}

Correspondence to: Dr Tomohiro Sugiyama, Department of Surgery, Toyooka Hospital, 1094 Tobera, Toyooka, Hyogo 668-0065, Japan

E-mail: t_sugiyama1103@yahoo.co.jp

Abbreviations: IGC, incidental gallbladder carcinoma; PTGBD, percutaneous transhepatic gallbladder drainage; (DIC)-CT, drip infusion cholecystocholangiography; MRCP, magnetic resonance cholangiopancreatography; LC, laparoscopic cholecystectomy; OC, open abdominal cholecystectomy; US, ultrasonography; CT, contrast-enhanced computed tomography; PE, papillary expanding type; FE, flat expanding type; NI, nodule infiltrating type; FI, flat infiltrating type; Fi, filling type; Gem, Gemzar; CDDP, cisplatin

Key words: incidental gallbladder carcinoma, additional resection, long-term outcomes, pT3 tumor, pT2 tumors, cholecystectomy treatment history and risk factors for recurrence. Furthermore, long-term survival was observed in patients with pT2 and pT3 tumors who did not undergo additional resection. Recurrence was not associated with the extent of tumor invasion but may be associated with other histopathological findings, preoperative treatment history, and risk factors for recurrence. Furthermore, long-term survival was observed in patients with pT2 and pT3 tumors who did not undergo additional resection. Even if it is a progressive IGC case, appropriate preoperative treatment or cholecystectomy without persistence of the carcinoma cell, based on a preoperative image evaluation and a postoperative histopathological examination, may greatly influence the long-term prognosis of IGC.

\section{Introduction}

Incidental gallbladder carcinoma (IGC) is defined as unexpected malignancy that is identified in the surgical gallbladder specimen of a cholecystectomy performed for a benign diagnosis, such as cholecystolithiasis or acute cholecystitis (1). IGC is diagnosed in $0.3-1.5 \%$ of all cholecystectomies; however, the frequency is higher in elderly patients, particularly those aged $\geq 80$ years, with a rate of $9 \%(2-5)$.

Gallbladder carcinoma is known as one of the very poor-prognosis cancers, It is necessary to be operated on not to make any dispersal and persistence of the tumor cells. Especially in advanced gallbladder carcinoma, the extended operation with the lymph node dissection is required. Of course, pre-operative diagnosis of the IGC is important to perform necessary operations, but the diversity of naked eye shape of the gallbladder carcinoma makes it difficult to diagnose in preoperative examination. Sometime we find it difficult to obtain the preoperative diagnosis of the cancer under cover of inflammatory disorder such as the cholecystitis. It is expected that the prognosis of the patients who underwent insufficient surgery is poorer than that of gallbladder carcinoma patients.

In some patients with IGC, preoperative percutaneous trans-hepatic gallbladder drainage (PTGBD) causes dispersal of tumor cells into the liver bed or the fistula of the drainage tube, which can result in local recurrence (6-8).

Previous studies have suggested that additional resection should be performed in progressive IGC with subserosal invasion of the gallbladder (pT2 and pT3) (9-12). However, there are often valid clinical reasons to defer reoperation for additional 
resection, particularly in elderly patients. In particular, in the local area where medical resources are limited and which has relatively high age composition, additional resection with the major involvement is hard to provide and our hospital has such a difficulty, too.

Thus, this study aimed to examine patients with IGC at the hospital to determine long-term outcomes and prognostic factors associated with recurrence. In the present study, although some IGC cases had subserosal tumor invasion (pT2 and pT3), a relatively good prognosis was observed. Preoperative treatment and pathological findings which suggest persistence of the tumor seemed to be connected with the recurrence.

\section{Materials and methods}

The medical records of 678 patients who underwent cholecystectomy at Toyooka Hospital (Hyogo, Japan), from September 2011 to November 2017 were reviewed. Preoperative evaluation included blood chemistry examination, ultrasonography (US), and contrast-enhanced computed tomography (CT) imaging. In several cases, a biliary evaluation by drip infusion cholecystocholangiography (DIC)-CT, magnetic resonance cholangiopancreatography (MRCP), or PTGBD was performed unless precluded by the need for emergent surgery.

At the hospital, the treatment of choice for surgical gallbladder disease is laparoscopic cholecystectomy (LC); open abdominal cholecystectomy (OC) is utilized when severe adhesions, hyperplasia or chronic inflammation of the gallbladder wall, Mirizzi syndrome, or gallbladder cancer are suspected based on the preoperative examination. Rapid bile cytological testing or pathological tissue diagnosis was not performed during cholecystectomy unless gallbladder carcinoma was strongly suspected preoperatively. Cases with IGC were defined as those in which gallbladder carcinoma was unlikely based on preoperative examination, with malignancy identified on postoperative histopathological examination. Cases in which gallbladder carcinoma was suspected preoperatively and extended cholecystectomy was performed (gallbladder resection with liver bed resection or lymph node dissection) were excluded. Patient and histopathological characteristics, surgical details, long-term outcomes, and factors associated with recurrence in the cases with IGC were examined.

\section{Results}

Twelve patients with IGC were identified from the 687 who underwent cholecystectomy during the study period, accounting for $54.5 \%(12 / 22)$ of all cases with postoperative histopathological diagnosis of gallbladder carcinoma. Table I presents the 12 cases with IGC, showing preoperative characteristics and surgical details. Corresponding histopathological details, treatments received, outcomes, and follow-up periods for each case are shown in Table II.

Patients with IGC tended to be older than those in the entire cohort of 678 patients who underwent cholecystectomy; median ages were 80 (range 70-89) years and 68 (range 16-97) years, respectively. Sex ratio was 7:5 (male:female). Ten patients with IGC underwent elective cholecystectomy performed because of a diagnosis of cholecystolithiasis; the other 2 patients underwent emergent surgery for acute cholecystitis. In 3 patients, PTGBD had been performed preoperatively. LC was performed in 7 patients, and OC was performed in 5. Hyperplasia of the gallbladder wall or pericystic inflammatory findings was identified in all cases except for one (case 1).

Obvious cholerrhagia in the abdominal cavity, including perioperative gallbladder puncture drainage, was observed in 4 cases.

The extent of tumor invasion observed pathologically in the surgical specimen was as follows: pT1a (1 case), pT1b (5 cases), pT2 (5 cases), and pT3 (1 case). Subserosal tumor invasion (pT2 and pT3) was observed in half of the cases.

Except in cases 5, 6, and 11, malignant cells were not detected at the liver bed and in the cystic duct stumps of the specimen. Microvascular invasion was found in 4 patients with pT2 (cases 3, 4, 8, and 12) and one with pT1b (case 6).

All patients with subserosal tumor invasion (pT2) were offered a reoperation for additional resection of the liver bed and dissection of the liver and duodenal ligament lymph nodes; however, only 1 patient (case 12) elected to proceed. Long-term postoperative oncological follow-up was achieved in 8 of the 11 patients who did not undergo additional resection: Cases 7 and 11 died 8 days and 1 month postoperatively, respectively, and case 10 relocated after the operation and was lost to follow-up. Postoperative recurrence of gallbladder carcinoma was observed in cases 4,6 , and 9 .

In case 4 , the macroscopic type of the tumor was nodular infiltrating (NI). Histopathologically, tumor invasion was graded pT2b (subserosal), and metastasis was observed in a \#12c lymph node incidentally attached to the neck of the resected gallbladder specimen. The patient declined additional resection and chemotherapy because of advanced age (81 years). Obstructive jaundice with porta hepatis lymphadenopathy occurred 22 months postoperatively, and she died 2 months later.

In case 6 , the macroscopic type of tumor was flat infiltrating (FI), with tumor invasion graded pT1b (muscularis propria) on histopathological examination. The surgical margin from the cystic duct was $<5 \mathrm{~mm}$, and additional resection was strongly recommended because invasion of the cystic duct stump was suspected. However, the patient opted for postoperative systemic chemotherapy and received gemcitabine $1,000 \mathrm{mg} / \mathrm{m}^{2}$ on days 1 and 8 of a 3 -week cycle. Four months after cholecystectomy, CT imaging revealed local recurrence around the cystic duct stump. Chemotherapy was continued; however, the regimen was changed to S-1 $80 \mathrm{mg} /$ day for days 1-14 of a 3-week cycle. Twenty-three months after cholecystectomy, obstructive jaundice because of direct invasion of the porta hepatis occurred, and he died 3 months later.

In case 9, PTGBD was performed prior to cholecystectomy. The macroscopic type of tumor was flat expanding (FE). Histopathological examination showed pT1b (muscularis propria) tumor invasion, with no tumor cells in the bile duct or liver bed stumps; there was no microvascular invasion. Postoperatively, he declined additional resection. Seven months after cholecystectomy, local recurrence in the liver bed stump was observed on CT imaging, and chemotherapy with gemcitabine, CDDP, and S-1 was initiated; he died 14 months after cholecystectomy. 

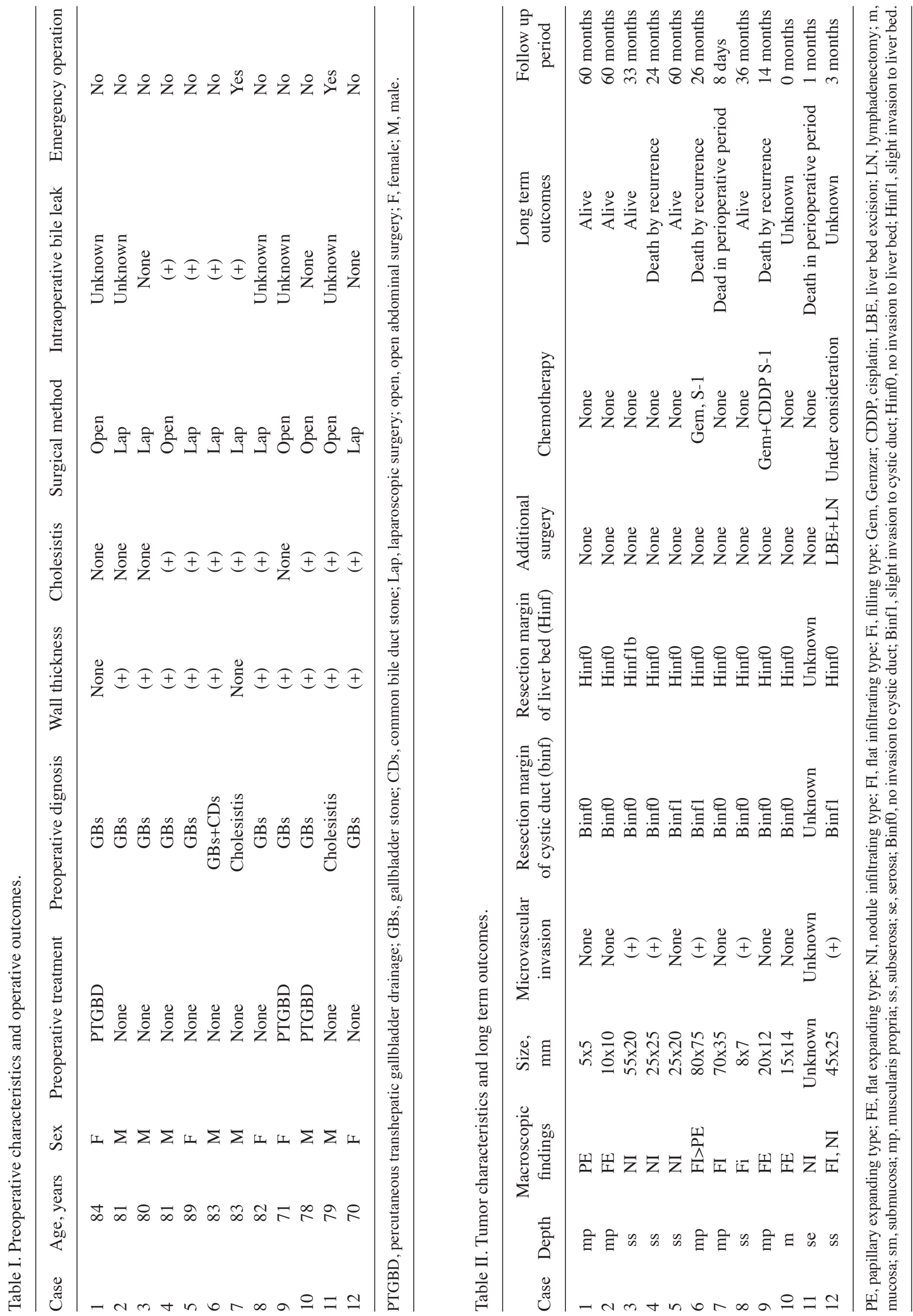


\section{Discussion}

IGC is diagnosed in $0.3-1.5 \%$ of all cholecystectomies; however, the frequency is higher in elderly patients, particularly those aged $\geq 80$ years, with a rate of $9 \%$ (9-12). Despite an appropriate evaluation, the preoperative diagnosis of gallbladder carcinoma can be difficult in patients with coexisting benign and malignant tumors, despite using advanced imaging techniques, and may not be suspected prior to surgery (13). In this report, IGC was discovered in $1.8 \%$ $(12 / 678)$ of the cholecystectomies performed during the study period. Although 22 patients with histopathologically proven gallbladder carcinoma were reviewed, the diagnostic accuracy of the preoperative evaluation was only $45 \%$ (10/22). After examining the resected gallbladder specimens, hyperplasia of the gallbladder wall, presence of cholecystitis, and varying macroscopic type (particularly the flat type) may cause difficulty in identifying gallbladder carcinoma preoperatively. In addition, there is often little time to complete a thorough preoperative evaluation in patients who require an emergent operation.

Here, in the cases with IGC, the macroscopic type of tumor varied. The NI type was observed in the pT2b (subserosal) and pT3 tumors in cases 3, 4, 5, 11, and 12; the nodular component tended to increase with increasing tumor invasion. In the pT1 (muscularis and muscularis propria) tumors, FI or FE types were observed (cases 2, 6, 7, 9, and 10). With regard to the invasive gallbladder cancers, it was difficult to suspect their presence upon macroscopic inspection of the surgical specimens.

Preoperative biliary cytological analysis may increase the accuracy of detecting gallbladder carcinoma preoperatively. Its utility following endoscopic nasogallbladder drainage (ENGBD) has been previously reported (14). In this study, PTGBD was performed as preoperative treatment in 3 patients (cases 1,9, and 10); unfortunately, gallbladder carcinoma was not suspected and biliary analysis was not performed. Notably, case 9 showed recurrence in the liver bed along the PTGBD fistula; procedure-related bile dispersal to the liver bed may have caused the recurrence. Other reported PTGBD-related recurrences include metastases to the skin through a fistula and peritoneal dissemination, where the resection both the liver bed and fistula to prevent recurrence is recommended (6-8). Therefore, ENGBD is preferable to PTGBD; if PTGBD is required, cytological analysis may be necessary preoperatively to decide the operative method and extent of resection (cholecystectomy or expanded cholecystectomy).

Reoperation for additional resection in patients with IGC discovered after cholecystectomy remains controversial. Some reports show that additional resection in cases with invasive IGC (pT2 or pT3) improves long term outcomes (15-21). Although degree of tumor differentiation $(16,17)$, microvascular invasion $(19,20)$, and depth of subserosal invasion (21) have been reported to be risk factors for recurrence, there are no well-established criteria for additional resection. Furthermore, gallbladder cancer with lymph node metastases, despite R0 resection, has a poor prognosis $(15,18)$. The effect of additional resection on long-term outcomes in patients with IGC remains unclear. The clinical decision for additional resection should be considered carefully on an individual basis.
In the present study, additional resection was not performed in 11 of the 12 patients with IGC, and 8 of these patients had long-term follow-up. Within 33 months, 5 of the 8 cases had no recurrence, whereas 3 cases (cases 4, 6, and 9) had recurrence during the follow-up period. No significant tendency was recognized toward recurrence based on histopathological findings or surgical details; however, there are notable facts regarding the 3 individual patients with recurrence. In case 4, a pT2 tumor with a lymph node metastasis attached to the cystic duct that recurred in the porta hepatis lymph nodes was revealed on postoperative pathological examination. The prediction of the recurrence pattern may be conducted by examining lymphatic flow. In case 6 , a pT1b tumor with tumor cells found in contiguity with $<5 \mathrm{~mm}$ of the cystic duct stump was revealed. Microinvasion of the cystic duct stump was suspected, and local recurrence was later observed there. In case 9, a pT1b tumor was revealed, and PTGBD was performed before cholecystectomy. Biliary dispersal along the liver bed and fistula of the drainage tube was suspected, and local recurrence at the liver bed was observed later. All 3 patients had risk factors for recurrence, and the area of recurrence could be explained pathologically; lymph node metastasis, biliary exposure, and microinvasion of the cystic duct stump appeared to play a critical role in recurrence. This suggests that the extent of additional resection should be based on the pathological findings or preoperative treatment in each individual case. For example, in case 9, additional resection of the liver bed and PTGBD fistula may be adequate to prevent recurrence.

Long-term outcomes in patients who did not receive additional resection in this study appear to be better than those previously reported (16). Five of 8 patients remain recurrence-free at a minimum of 33 months, and 3 of 8 had invasive tumors (pT2).

Of course, there are a few number of cases in this study, and further examination is necessary, What we want to emphasize most is that, presence of an appropriate preoperative treatment or cholecystectomy without persistence of the cancer cell, based on a preoperative image evaluation and a postoperative histopathological examination may greatly influence the long-term prognosis of IGC.

It may be necessary to investigate more patients with a higher recurrence risk, and include more patients who do not undergo additional resection because this scenario is often encountered in clinical practice. IGC is relatively frequent in patients aged $>80$ years, and additional resection is often not a viable option because of various factors such as poor performance status, comorbidities, and lack of motivation. Therefore, even if it was a progressive IGC cases, to perform appropriate preoperative treatment and cholecystectomy, and carefully choice of additional resection by the pre and post-operative examination may be most important for the prognosis of IGC cases.

\section{Acknowledgements}

Not applicable.

\section{Funding}

No funding was received. 


\section{Availability of data and materials}

All data generated or analyzed during this study are included in this published article.

\section{Authors' contributions}

TS conceived and designed the study. TS, KM, YF, HK, AM, SU and MT were in charge of treatment for individual cases and cooperated in the accumulation and analysis of the data. YA investigated and analyzed the pathological data. All authors read and approved the final manuscript.

\section{Ethics approval and consent to participate}

The requirement for patient consent was waived. The authors obtained Ethical Approval from the Ethics Committee of Toyooka Hospital (Hyogo, Japan).

\section{Patient consent for publication}

The Ethics Committee of Toyooka Hospital obtained approval from each patient to manage individual details for appropriate use. The authors also made efforts to remove any identifying information to protect the privacy of the patients.

\section{Competing interests}

The authors declare that they have no competing interests.

\section{References}

1. Varshney S, Buttirini G and Gupta R: Incidental carcinoma of the gallbladder. Eur J Surg Oncol 28: 4-10, 2002.

2. Choi SB, Han HJ, Kim CY, Kim WB, Song TJ, Suh SO, Kim YC and Choi SY: Incidental gallbladder cancer diagnosed following laparoscopic cholecystectomy. World J Surg 33: 2657-2663, 2009

3. Ouchi K, Mikuni J and Kakugawa Y; Organizing Committee, The 30th Annual Congress of the Japanese Society of Biliary Surgery: Laparoscopic cholecystectomy for gallbladder carcinoma: Results of a Japanese survey of 498 patients. J Hepatobiliary Pancreat Surg 9: 256-260, 2002.

4. Kwon AH, Imamura A, Kitade H and Kamiyama Y: Unsuspected gallbladder cancer diagnosed during or after laparoscopic cholecystectomy. J Surg Oncol 97: 241-245, 2008.

5. Tian YH, Ji X, Liu B, Yang GY, Meng XF, Xia HT, Wang J, Huang ZQ and Dong JH: Surgical treatment of incidental gallbladder cancer discovered during or following laparoscopic cholecystectomy. World J Surg 39: 746-752, 2015.

6. Yamakawa T, Itoh S, Hirosawa K, Miyoshi T, Katoh K, Iizumi S and Kawabata K: Seeding of gallbladder carcinoma along the tract after percutaneous transhepatic choledochoscopy. Am J Gastorntel 79: 649-651, 1983.
7. Kim WS, Barth KH and Zinner M: Seeding of pancreatic carcinoma along the transhepatic catheter tract. Radiology 143: 427-428, 1982

8. Cutherell L, Wanebo HJ and Tegtmeyer CJ: Catheter tract seeding after percutaneous biliary drainage for pancreatic cancer. Cancer 57: 2057-2061, 1983.

9. Goetze TO and Paolucci V: Benefits of reoperation of T2 and more advanced incidental gallbladder carcinoma: Analysis of the German registry. Ann Surge 247: 104-108, 2008.

10. Goetze TO and Paolucci V: Adequate extent in radical re-resection of incidental gallbladder carcinoma: Analysis of the German Registry. Surg Endosc 24: 2156-2164, 2010.

11. Foster JM, Hoshi H, Gibbs JF, Iyer R, Javie M, Chu Q and Kuvshinoff B: Gallbladder cancer: Defining the indications for primary radical resection and radical re-resection. Ann Surg Oncol 14: 833-840, 2007.

12. Braghetto I, Bastias J, Csendes A, Chiong H, Compan A, Valladares H and Rojas J: Gallbladder carcinoma during laparoscopic cholecystectomy: Is it associated with bad prognosis? Int Surg 84: 344-349, 1999.

13. Inui K, Yoshino J and Miyoshi H: Diagnosis of gallbladder tumors. Intern Med 50: 1133-1136, 2010.

14. Itsuki H, Serikawa M, Sasaki T, Ishii Y, Tsushima K, Furukawa Y, Murakami Y, Arihiro K and Chayama K: Indication and usefulness of bile juice cytology for diagnosis of gallbladder cancer. Gastroenterol Res Pract 2018: 5410349, 2018.

15. Shimada H, Endo I, Togo S, Togo S, Nakano A, Izumi T and Nakagawara G: The role of lymph node dissection in the treatment of gallbladder carcinoma. Cancer 79: 892-899, 1997.

16. Choi SB, Han HJ, Kim CY, Kim WB, Song TJ, Suh SO, Kim YC and Choi SY: Surgical outcomes and prognostic factors for T2 gallbladder cancer following surgical resection. J Gastrointest Surg 14: 668-678, 2010

17. Ito $\mathrm{H}$, Ito $\mathrm{K}$, D'Angelica M, Gonen M, Klimstra D, Allen P, DeMatteo RP, Fong Y, Blumgart LH and Jarnagin WR: Accurate staging for gallbladder cancer: Implications for surgical therapy and pathological assessment. Ann Surg 254: 320-325, 2011.

18. Negi SS, Singh A and Chaudhary A: Lymph nodal involvement as prognostic factor in gallbladder cancer: Location, count or ratio? J Gastrointest Surg 15: 1017-1025, 2011.

19. Shibata K, Uchida H, Iwaki K, Kai S, Ohta M and Kitano S: Lymphatic invasion: An important prognostic factor for Stages T1b-T3 gallbladder cancer and an indication for additional radical resection of incidental gallbladder cancer. World J Surg 33: 1035-1041, 2009.

20. Yamaguchi R, Nagino M, Oda K, Kamiya J, Uesaka K and Nimura Y: Perineural invasion has a negative impact on survival of patients with gallbladder carcinoma. Br J Surg 89: 1130-1136, 2002.

21. Wakai T, Shirai Y, Yokoyama N, Ajioka Y, Watanabe H and Hatakeyama K: Depth of subserosal invasion predicts long-term survival after resection in patients with T2 gallbladder carcinoma. Ann Surg Oncol 10: 447-454, 2003. 